\title{
Polyisobutylene: A Most Unusual Polymer
}

\author{
K. KUNAL, ${ }^{1}$ M. PALUCh, ${ }^{2}$ C. M. ROLAND, ${ }^{3}$ J. E. PUSKAS, ${ }^{1}$ Y. CHEN, ${ }^{1}$ A. P. SOKOLOV ${ }^{1}$ \\ ${ }^{1}$ Department of Polymer Science, The University of Akron, Akron, Ohio 44325-3909 \\ ${ }^{2}$ Institute of Physics, Silesian University, ulica Uniwersytecka 4, 40-007 Poland \\ ${ }^{3}$ Naval Research Laboratory, Chemical Division, Code 6120, Washington, District of Columbia 20375-5342
}

Received 22 February 2008; revised 7 April 2008; accepted 10 April 2008

DOI: 10.1002 / polb.21473

Published online in Wiley InterScience (www.interscience.wiley.com).

\begin{abstract}
The influence of molecular weight, $M$, on the fragility and fast dynamics in polyisobutylene (PIB) was studied using dielectric and mechanical relaxation spectroscopies, calorimetry, and Raman spectroscopy. The measurements indicate a decrease in fragility with increasing $M$ for shorter chains, in the range of $M$ where $T_{\mathrm{g}}$ is $M$-dependent. Such behavior is not observed for other polymers and is at odds with traditional theoretical models that predict an increase in fragility with chain length. These results confirm the unusual character of PIB, as evident in various properties including extremely low gas permeability, a low fragility, and a segmental relaxation spectrum much broader than expected for a low-fragility material. The reason for this anomalous behavior remains unclear, but might be related to the symmetric structure of the PIB repeat unit, together with comparable flexibility of both structural components, the backbone and side groups. () 2008 Wiley Periodicals, Inc. J Polym Sci Part B: Polym Phys 46: 1390-1399, 2008

Keywords: dielectric relaxation; fragility; glass transition; light scattering; mechanical spectroscopy; polyisobutylene; Raman spectroscopy; structure-property relationship
\end{abstract}

\section{INTRODUCTION}

Polyisobutylene (PIB) is a commercially important polymer that finds a great number of applications exploiting its thermal stability, good flexibility at ambient temperature, and impermeability to gases. Reflecting this importance, in 1949 the National Bureau of Standards (currently the National Institute of Standards and Technology) chose PIB as a standard polymer to establish "usual polymeric" properties. Twentyseven laboratories worldwide collaborated in a study of a PIB sample, with the accumulated results used to define what was assumed to be the standard viscoelastic properties of polyedu).

Correspondence to: A. P. Sokolov (E-mail: alexei@uakron.

Journal of Polymer Science: Part B: Polymer Physics, Vol. 46, 1390-1399 (2008)

○ 2008 Wiley Periodicals, Inc. mers. ${ }^{1,2}$ This early work on PIB played a central role in establishing general polymeric properties, for example, the molecular weight dependence of the viscosity of entangled polymers and the time-temperature superposition principle.

However, it appears that PIB has many properties that differentiate it from most of the other polymers: (i) a markedly low permeability to small molecule penetrants ${ }^{3}$; (ii) one of the weakest temperature dependences of structural relaxation and viscosity (i.e., low fragility) ${ }^{4,5}$ and, correspondingly, strong inelastic scattering (boson peak) even at temperatures much above the glass transition ${ }^{6}$; (iii) a very small difference between the temperature dependences of segmental and chain relaxations, ${ }^{4,5}$ which can lead to an apparent conformance to time-temperature superpositioning, unlike the obvious breakdown seen in other polymers such as polystyrene, polyvinyl acetate, and polypropylene ${ }^{5,7,8}$; 
(iv) a mechanical segmental dispersion much broader than expected for such a low fragility material ${ }^{8}$; and (v) a very unusual spectrum of the fast dynamics; that is, a "constant loss" regime where the susceptibility changes negligibly with frequency. ${ }^{9}$ These examples illustrate that PIB can be viewed as a very unusual polymer with properties deviating from behavior common for many other polymers.

The glass transition in supercooled liquids is associated with rapid changes of the rate of structural relaxation (segmental relaxation process in polymers). The segmental relaxation time, $\tau_{\alpha}$, increases by many orders of magnitude upon cooling only a few degrees at temperatures close to the glass transition temperature, $T_{\mathrm{g}} \cdot \tau_{\alpha}$ in polymers exhibits a strongly non-Arrhenius temperature dependence, which is well-approximated by the Vogel-Fulcher-Tamman (VFT) equation as follows:

$$
\tau_{\alpha}=\tau_{0} \exp \left[B /\left(T-T_{0}\right)\right]
$$

where $\tau_{0}$ and $B$ are material constants and $T_{0}$ is the temperature of an "ideal" glass transition. The concept of fragility has been introduced to characterize the steepness of the temperature variations of $\tau_{\alpha}$ close to $T_{\mathrm{g}}$. Essentially, fragility characterizes the degree of deviation of the temperature dependence of $\tau_{\alpha}$ from Arrhenius-like behavior (i.e., deviation from thermally activated behavior with a constant activation energy). A "strong" material has a near Arrhenius temperature dependence of $\tau_{\alpha}$, whereas a "fragile" material exhibits strongly non-Arrhenius behavior. To quantify the fragility, Boehmer et al. ${ }^{10}$ defined the fragility (steepness) index $m$ as follows:

$$
m=\left.\frac{\partial \log \tau_{\alpha}}{\partial\left(T_{\mathrm{g}} / T\right)}\right|_{T=T_{\mathrm{g}}} .
$$

In general, polymers tend to be more fragile than molecular liquids. It has been observed that polymers with a rigid backbone or bulky pendant groups, such as polystyrene (PS) and poly(methyl methacrylate) (PMMA), exhibit a strong increase in fragility with increase in molecular weight. ${ }^{11,12}$ On the other hand, flexible polymer chains such as poly(dimethyl siloxane) (PDMS) and poly(methyl phenyl siloxane) (PMPS) show less or no increase in fragility with molecular weight. ${ }^{13}$ These results were explained in the framework of the coupling model $^{12}$ and more recently in the model of Dudowicz et al. ${ }^{14}$ The common idea of both approaches is that fragility relates to the severity of steric constraints from neighboring segments, which impede segmental motions ${ }^{12}$ and/ or frustrate chain packing. ${ }^{14}$ The packing idea leads to the expectation that packing of rigid chains or chains with bulky side groups will be more frustrated as $M$ increases. As a result, such polymers should exhibit a strong increase in fragility with increasing molecular weight. Flexible chains should pack better and, according to the model ${ }^{14}$ should show a much weaker increase in fragility with $M$. No theory predicts a decrease of fragility with molecular weight.

However, preliminary studies of PIB presented in ref. 15 indicated a decrease in fragility with increase of the chain length. This would suggest that PIB again exhibits a trend opposite to the pattern of other polymers and to the theoretical expectation. We present herein a detailed study of the dynamics of PIB using mechanical and calorimetry measurements, dielectric relaxation, and Raman scattering spectroscopy. The results demonstrate a decrease of fragility with increase in molecular weight for PIB. Possible reasons for the unusual behavior of PIB are discussed.

\section{EXPERIMENTAL}

\section{Materials}

PIB samples were precision synthesized by living carbocationic polymerization in the Department of Polymer Science at the University of Akron. The polymerization of isobutylene was initiated by 2-chloro-2,4,4-trimethyl-pentane/ $\mathrm{TiCl}_{4}{ }^{16}$ and terminated using trimethyl aluminum (Aldrich) ${ }^{17}$ to obtain PIBs with only $\mathrm{C}$ and $\mathrm{H}$ atoms. ${ }^{1} \mathrm{H}$ NMR analysis of the resulting PIBs showed that they were exclusively terminated by $\mathrm{CH}_{3}$ groups (synthesis details will be reported elsewhere). This is important for our study to exclude any chain-end interactions. Four different molecular weight PIB samples (Table 1) were synthesized using this procedure.

\section{Dynamic Mechanical Analysis}

Dynamic mechanical data were obtained using a Bohlin VOR with a parallel plate geometry. Isothermal measurements were made over a $10-12{ }^{\circ} \mathrm{C}$ 


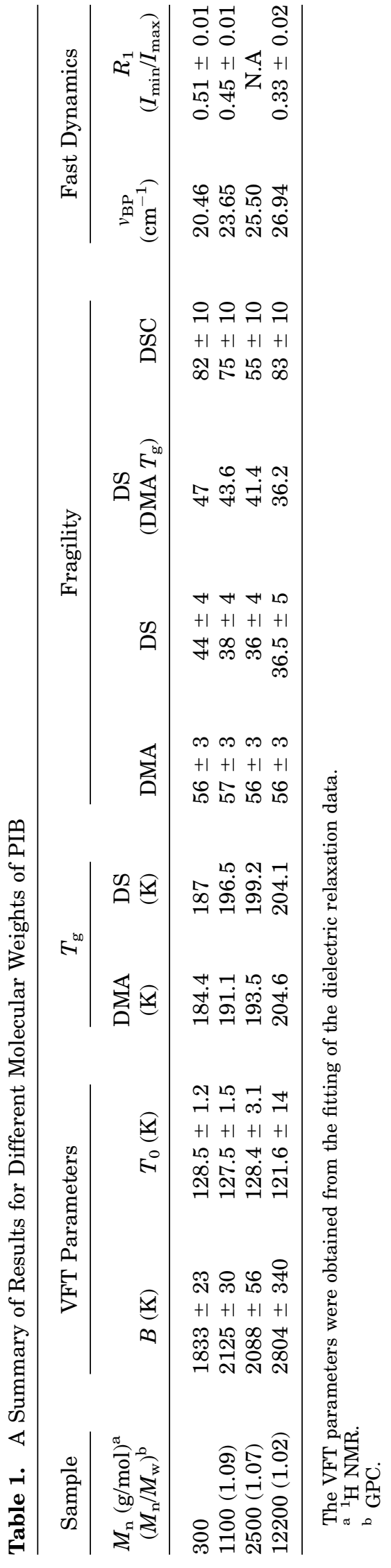

range of temperatures, corresponding typically to $0.96 \leq T_{\mathrm{g}} / T \leq 1.01$, with $0.1 \mathrm{~K}$ control. The frequency was varied according to the temperature within the range $0.0001-2 \mathrm{~Hz}$ to obtain the peak in the loss modulus, $G^{\prime \prime}$, within the measurement window. Strains were between 0.02 and $0.5 \%$. Figure 1(a) presents $G^{\prime \prime}$ spectra for the sample with smallest molecular weight.

\section{Rate Dependent DSC}

Differential scanning calorimetry (DSC) was carried out using TA Instruments Q100 with liquid nitrogen cooling. Samples were cooled from the liquid state to $50{ }^{\circ} \mathrm{C}$. below $T_{\mathrm{g}}$, at rates, $q_{\mathrm{c}}$, from 0.1 to $10 \mathrm{~K} / \mathrm{min}$. After $5 \mathrm{~min}$, this was followed by heating at $10 \mathrm{~K} / \mathrm{min}$ through $T_{\mathrm{g}}$. Fictive temperatures were calculated from the heating data.

\section{Dielectric Spectroscopy}

The dielectric measurements in the frequency range $0.1 \mathrm{~Hz}-10 \mathrm{MHz}$ were carried out on two spectrometers, both using the Novocontrol Alpha analyzer, with the complex dielectric permittivity $\varepsilon^{*}(\omega)=\varepsilon^{\prime}(\omega)-\varepsilon^{\prime \prime}(\omega)$ obtained from samples in a parallel-plate capacitor geometry. For one set of experiments, temperature was controlled using a Novocontrol Quattro temperature control unit; the other set utilized a closed-cycle helium cryostat. In both cases, the stability was at least $0.1 \mathrm{~K}$. Measurements below $0.1 \mathrm{~Hz}$ were not attempted because PIB has a very low dipole moment and thus weak dielectric signal. This problem becomes significantly worse with increasing molecular weight. For this reason, dielectric relaxation times were determined only up to $\tau_{\alpha} \sim 1 \mathrm{~s}$. Figure 1(b) presents the dielectric loss spectra for the PIB sample with the smallest molecular weight.

\section{Raman Spectroscopy}

Depolarized Raman scattering spectra were measured in back-scattering geometry using a Jobin Yvon T64000 triple monochromator, an $\mathrm{Ar}^{+}$ion laser with a wavelength of $514.5 \mathrm{~nm}$ and laser power $\sim 15 \mathrm{~mW}$ at the sample. Samples were kept in silanized glass ampoules, placed into an optical cryostat (Oxford Instruments, Optistat). Measurements were carried out at $150 \mathrm{~K}$, that is, below $T_{\mathrm{g}}$ for all samples. Depolarized light scattering spectra of PIB sam- 

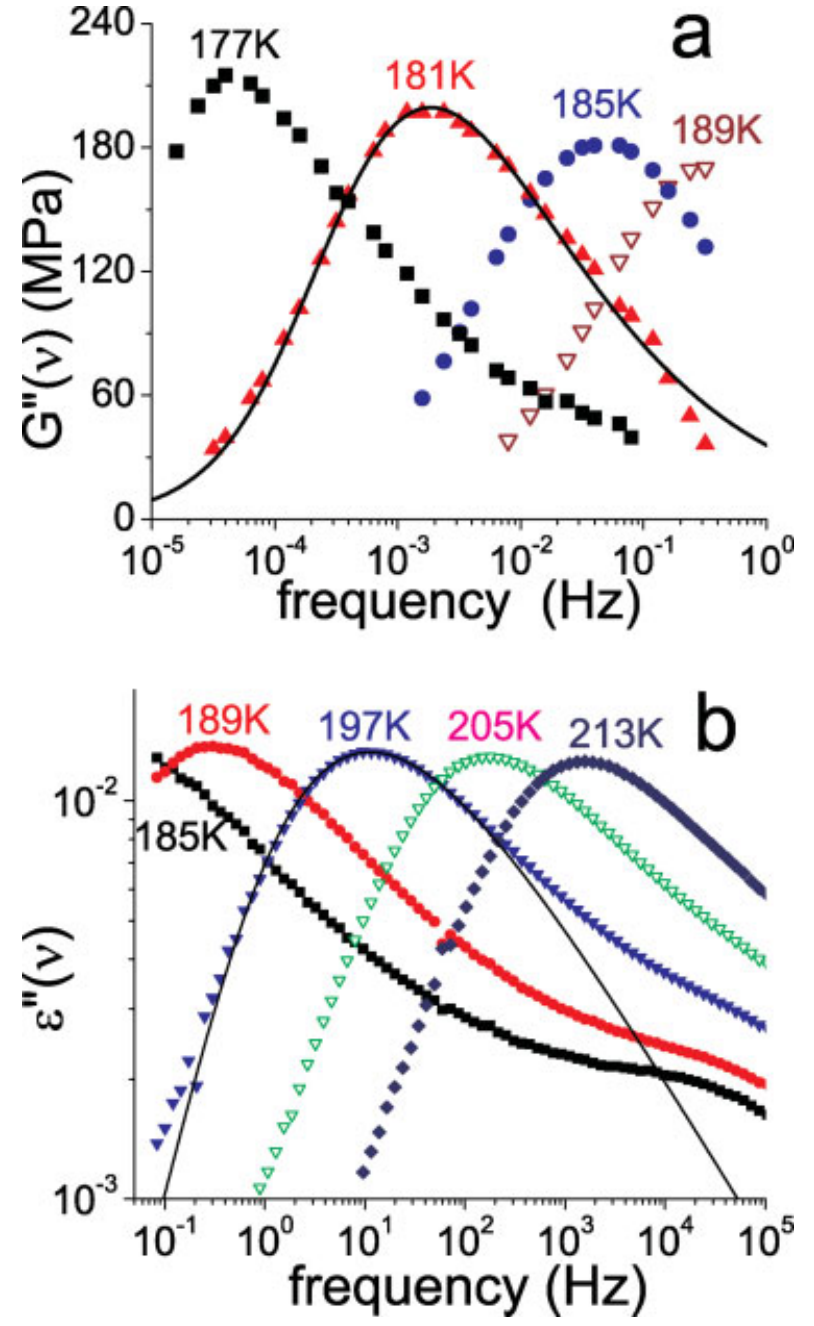

Figure 1. Relaxation spectra of PIB, $M=300 \mathrm{~g} /$ mol: (a) mechanical loss modulus on a linear ordinate scale and (b) dielectric loss spectra on a logarithmic scale. The solid line in both figures is the transform of the KWW function with $\beta=0.42$.

ples with various molecular weights are presented in Figure 2, as the spectral density $I_{n}$ $=I /[v(n(v)+1)]$, where $n(v)=[\exp (h v / k T)-1]^{-1}$ is the temperature Bose factor. The intensity of the spectra was scaled at the optical mode around $225 \mathrm{~cm}^{-1}$.

\section{RESULTS}

\section{Local Segmental Dispersion}

Figure 1 shows the mechanical and dielectric loss spectra for PIB with $M=300 \mathrm{~g} / \mathrm{mol}$. The loss peaks correspond to local segmental relaxation. Fits of the spectra to the Fourier transform of the Kohlrausch-Williams-Watts function (KWW) are included,

$$
f(t) \sim \exp \left[-\left(t / \tau_{\mathrm{KWW}}\right)^{\beta}\right]
$$

where $\beta$ is the KWW exponent describing the breadth of the relaxation function and the KWW relaxation time, $\tau_{\mathrm{KWW}}<\tau_{\alpha}$ (the latter defined from the peak frequency), reflecting the skew toward higher frequencies in $G$ " and $\varepsilon "$. The $\beta$ $=0.42$, obtained for both spectroscopies, is essentially independent of temperature in the range studied.
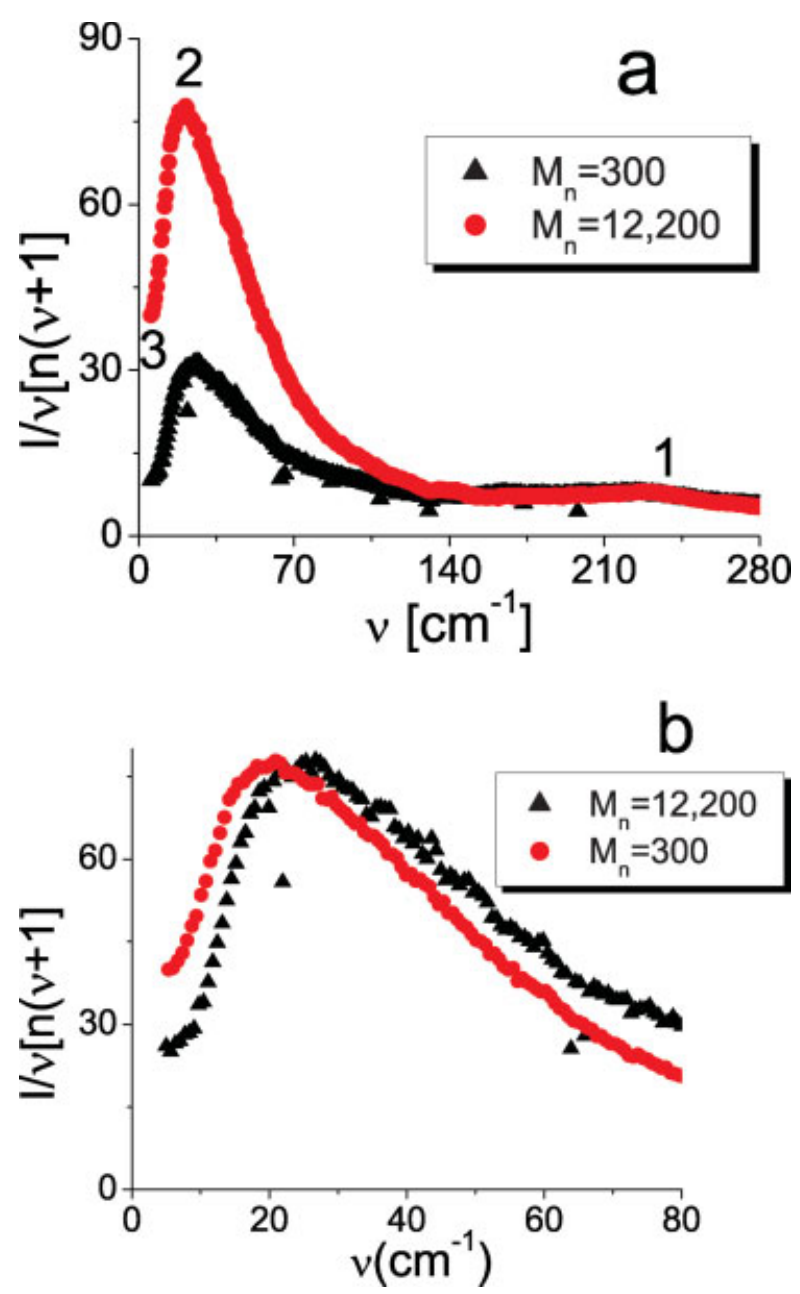

Figure 2. Depolarized light scattering spectra of PIB with different molecular weights presented as the spectral density at $T=150 \mathrm{~K}$. (a) Intensity normalized at the optical mode $v \sim 220 \mathrm{~cm}^{-1}$. A strong decrease of the boson peak intensity with molecular weight is evident. (b) Intensity normalized at the boson peak maximum, revealing the shift in peak position to higher frequencies with increase in molecular weight.

Journal of Polymer Science: Part B: Polymer Physics DOI $10.1002 /$ polb 


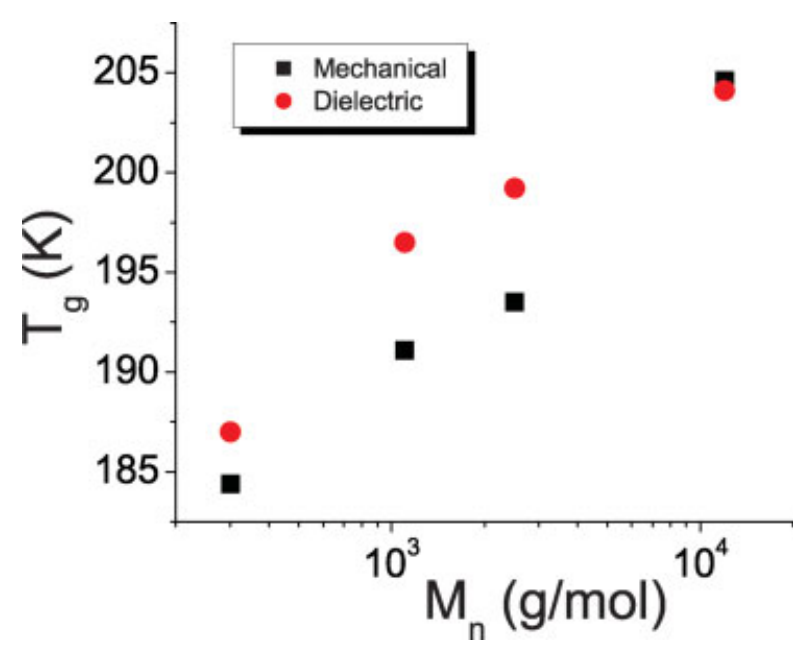

Figure 3. Glass transition temperature versus molecular weight of PIB measured using mechanical (squares) and dielectric (circles) relaxation. [Color figure can be viewed in the online issue, which is available at www.interscience.wiley.com.]

\section{Estimate of the Glass Transition Temperature}

The characteristic relaxation time, $\tau_{\alpha}$, was obtained as the reciprocal of the frequency of the maximum of the segmental relaxation process in $\varepsilon^{\prime \prime}(v)$ or in $G^{\prime \prime}(v)$, using $\tau_{\alpha}=\left(2 \pi v_{\max }\right)^{-1}$. Conventionally, $T_{\mathrm{g}}$ is defined as the temperature at which $\tau_{\alpha}=100 \mathrm{~s}$. However, we were not able to measure the dielectric spectra at low frequencies; therefore, we have chosen $T_{\mathrm{g}}$ as the temperature at which $\tau_{\alpha}=1 \mathrm{~s}$. Figure 3 presents the molecular weight dependence of the obtained glass transition temperatures. The mechanical relaxation times are shorter (corresponding to a lower $T_{\mathrm{g}}$ ) than dielectric $\tau_{\alpha}$ for most of the PIB samples. Figure 4 presents the temperature dependence of the segmental relaxation times for all samples as a function of $T_{\mathrm{g}} / T$.

\section{Estimate of Fragility Index, $m$}

The value of $T_{\mathrm{g}}=T\left(\tau_{\alpha}=1 \mathrm{~s}\right)$ was used in the calculation of the fragilities. Qualitative analysis of the data presented in Figure 4 shows that the temperature dependence of segmental relaxation in the dielectric experiment becomes steeper with decrease in $M$ for the three lowest molecular weight samples. The mechanical relaxation data do not show such a clear trend. Mechanical measurements, however, extend only down to $\tau$ $\sim 0.1 \mathrm{~s}$, whereas the dielectric data extend to much shorter relaxation times $\tau \sim 10^{-6}$ s (Fig. 4 , providing basis for more accurate analysis.
To estimate the steepness index, we fit the experimental data (Fig. 4) using the VFT equation (eq 1). Results of the fit of the dielectric data are presented in Table 1 . Substituting eq 1 into the eq 2, we obtain:

$$
m=\frac{B T_{\mathrm{g}}}{\left(T_{\mathrm{g}}-T_{0}\right)^{2} \ln 10}
$$

Since this analysis uses all the measured data, it is more precise than taking the slope at $T_{\mathrm{g}} . T_{\mathrm{g}}$ from mechanical and dielectric data differ somewhat (Fig. 3, Table 1) and we use both values of $T_{g}$ to estimate the fragility from dielectric data. The data show the same trend, decrease in fragility with molecular weight, regardless of the chosen $T_{\mathrm{g}}$. The error-bars of the fragility values have been estimated from the error-bars of the VFT fit parameters. Because the mechanical data above $T_{\mathrm{g}}$ are limited, we used a simple linear fit of the $\log \tau$ versus $T_{\mathrm{g}} / T$ to estimate the fragility. The error-bars for the fragility values were estimated from the error-bars of the linear fit.

The fragility was also determined by DSC measurements from the change in the fictive temperature, $T_{\mathrm{f}}$, with cooling rate, ${ }^{18}$

$$
m \equiv-\frac{d \log \left(q_{\mathrm{c}}\right)}{d\left(T_{\mathrm{f}, \mathrm{ref}} / T_{\mathrm{f}}\right)}
$$

where $T_{\mathrm{f}, \mathrm{ref}}$ is the fictive temperature for a cooling rate of $10 \mathrm{~K} / \mathrm{min}$. The error-bars for fragility

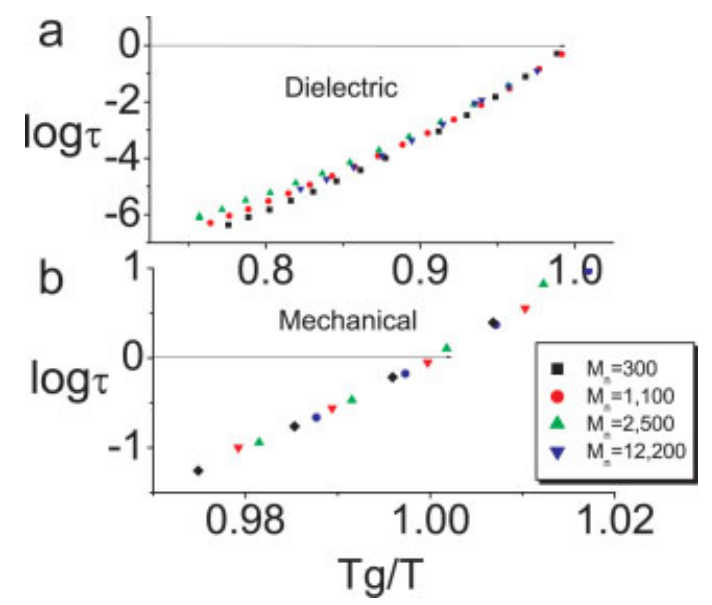

Figure 4. Segmental relaxation time versus $T_{\mathrm{g}} / T$ obtained from (a) dielectric measurements and (b) mechanical measurements. [Color figure can be viewed in the online issue, which is available at www.interscience.wiley.com.]

Journal of Polymer Science: Part B: Polymer Physics DOI 10.1002/polb 
values in this case have been estimated using traditional error propagation method. These calorimetric fragilities correspond to relaxation times on the order of $100 \mathrm{~s}^{18}$ The higher value of $\tau_{\alpha}\left(T_{\mathrm{g}}\right)$ in the DSC measurements, as expected, yields larger values of $m$ than the spectroscopic determinations. Still the obtained DSC estimates of the fragility value, especially at higher molecular weight, appear to be higher than usually reported in literature.

Analysis of the dielectric and DSC results shows that the steepness index decreases with molecular weight through $M \sim 2500 \mathrm{~g} / \mathrm{mol}$; however, this tendency is not apparent in the mechanical relaxation data (Fig. 5). For the highest molecular weight sample, $M=12,200 \mathrm{~g} / \mathrm{mol}$, which is approaching the high polymer limiting behavior (e.g., molecular weight independent $T_{\mathrm{g}}{ }^{19}$ ), the fragility becomes constant or might even increase. We emphasize that the choice of $\tau_{\alpha}\left(T_{\mathrm{g}}\right)=1 \mathrm{~s}$ leads to values of the fragility lower than determined at the conventionally defined $\tau\left(T_{\mathrm{g}}\right)=100 \mathrm{~s}$.

\section{Fast Dynamics}

The depolarized Raman spectra of PIB at $150 \mathrm{~K}$ have three characteristic features [Fig. 2(a)]: (1) An optical mode observed around $220 \mathrm{~cm}^{-1}$ arising from some microscopic vibrations; (2) the low-frequency "boson" peak at $v \sim 30 \mathrm{~cm}^{-1}$ arising from collective vibrations; and (3) Quasielastic scattering (QES) that appears at frequencies below $\sim 10 \mathrm{~cm}^{-1}$ and is usually ascribed to a fast (picosecond) relaxation.

Figure 2(b) shows the low-frequency part of the spectra normalized at the Boson peak intensity. A clear increase is observed in the boson peak frequency, $v_{\mathrm{BP}}$, with increase in molecular weight. This result is similar to earlier inelastic neutron scattering data presented in ref. 15. For a more quantitative analysis of the Boson peak frequency, the depolarized light scattering spectra were fit using the expression as follows:

$$
I_{\mathrm{n}}(v)=\frac{A v_{0}}{v_{0}^{2}+v^{2}}+B \exp \left\{\frac{-\left[\ln \left(v / v_{\mathrm{BP}}\right)\right]^{2}}{2\left(W / v_{\mathrm{BP}}\right)^{2}}\right\}
$$

Here the first term describes the quasi-elastic contribution approximated by a single Lorentzian with width $v_{0}$ and the second term represents the Boson peak approximated by a log-normal distribution with width $W$. Results of the fitting are presented in Table 1.

\section{DISCUSSION}

We note that the segmental relaxation spectra appear strongly stretched in both mechanical and dielectric measurements (Fig. 1). It is known that the breadth of the structural or segmental relaxation function of most glass-forming materials correlates with their fragility, although some exceptions have been reported. ${ }^{20}$ An approximate empirical relationship is $m$ $=250( \pm 30)-320 \beta .^{10}$ Using $\beta=0.42$ for PIB (Fig. 1) gives $m=116 \pm 30$. This is more than twofold larger than the measured value (Fig. $5)^{10}$ and illustrates another anomaly in the behavior of PIB. It is known that PIB has a secondary relaxation contributing to both dielectric and mechanical loss spectra; it is visible in our spectra at lower temperatures (Fig. 1). It is obvious, however, that this secondary process does not affect significantly the observed broadening of the segmental relaxation peak (Fig. 1).

There is substantial scatter in the values of fragility estimated using the different techniques (Fig. 5). Although mechanical spectroscopy suggests only weak or no $M$ dependence of fragility, more accurate dielectric measurements show a clear decrease in fragility with $M$ for molecular weights below the high polymer limiting value in agreement with the DSC measurements. The DSC results are also in agreement with earlier calorimetric estimates presented in ref. 15, which reported a significant decrease in fragility. We emphasize that the observed decrease in fragility with molecular weight occurs in the range where $T_{\mathrm{g}}$ also exhibits molecular weight dependence. This behavior of PIB is quite distinct from that known for other polymers and predicted by various models. ${ }^{14,21}$

Analysis of the fast dynamics also suggests that the fragility will decrease with increasing molecular weight. It has been shown that the ratio of the fast relaxation contribution (QES) to the Boson peak maximum, defined as the ratio of intensity at the minimum between QES and the Boson peak to the intensity at the Boson peak maximum $R_{1}=I_{\min } / I_{\max }$, correlates with the fragility of polymers; specifically, a decrease in $R_{1}$ usually indicates a decrease in fragility. ${ }^{22}$ The same is true also for nonpolymeric glass-forming systems. ${ }^{23}$ Although the nature of this correlation remains unclear, ${ }^{23,24}$ the fast dynamics usually provide a clear indication of fragility. $R_{1}$ has been found to increase with increase in $M$ for PS, along with its fragility, whereas it decreases with 


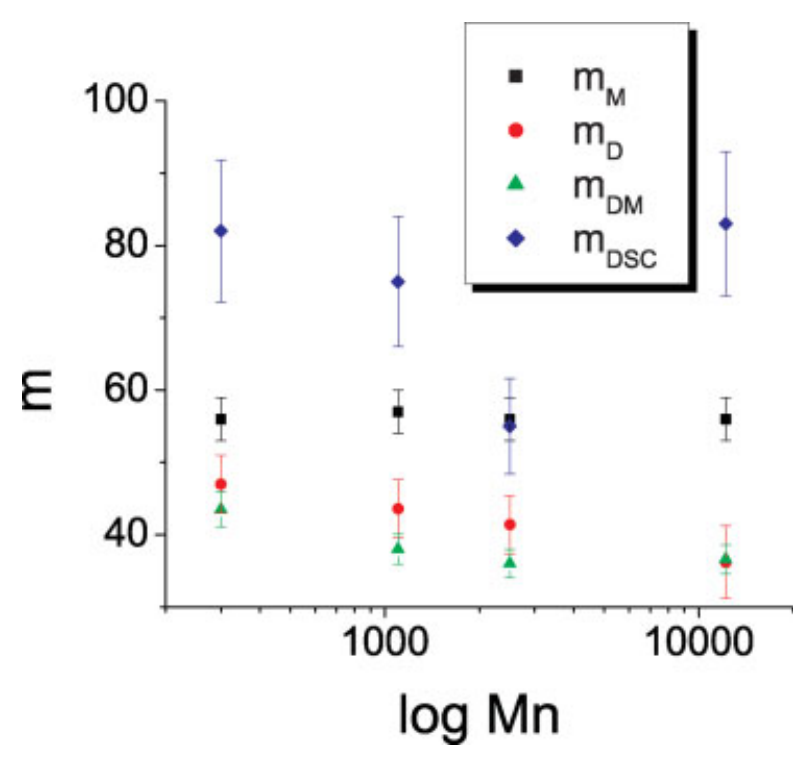

Figure 5. Molecular weight dependence of fragility in PIB estimated using different methods: $m_{\mathrm{M}}$ : mechanical relaxation using $T_{\mathrm{g}}$ from mechanical measurements; $m_{\mathrm{D}}$ : dielectric relaxation using $T_{\mathrm{g}}$ from dielectric measurements; $m_{\mathrm{DM}}$ : dielectric data but with $T_{\mathrm{g}}$ obtained from mechanical data; $m_{\mathrm{DSC}}$ : ratedependent DSC. [Color figure can be viewed in the online issue, which is available at www.interscience. wiley.com.]

increase in $M$ for PIB in neutron scattering experiments. ${ }^{15}$ In the case of neutron scattering in PIB, there is a possible complication from the contribution of the methyl group to the QES spectra: six out of eight hydrogen atoms (the main contributors to the neutron scattering) in the repeat unit of PIB are attached to the methyl side groups and the latter have rather low energy barriers to rotation. Methyl groups, however, do not contribute to the light scattering spectra. Nevertheless, we observe a decrease in $R_{1}$ with molecular weight in the light scattering data [Fig. 2(b), Table 1]. Thus, regardless of the methyl group contribution, $R_{1}$ decreases with $M$ in PIB, opposite to the trend observed in PS in ref. 15. Although the extension of general correlations of polymer behavior to a material such as PIB, which exhibits exceptional properties, may be problematic, it is nevertheless tempting to interpret the light scattering results as an additional indication that the fragility of PIB decreases with molecular weight, unlike the behavior of virtually all other studied polymers.

Figure 6 shows the molecular weight dependence of fragility reported for various polymers. Because of the higher accuracy of dielectric over mechanical measurements, the fragility values obtained from this technique were used for comparison with literature results. For $\mathrm{PS}^{25,26}$ and PMMA $^{10,14}$ fragility increases strongly with $M$, whereas the fragility of PDMS is essentially $M$ independent (Fig. 6). ${ }^{13,27}$ As noted in ref. 15, the fragility of many oligomers (short chains) falls in the range characteristic of molecular liquids with van-der-Waals interactions; that is, $m \sim$ 60-90 (shaded region). With the increase of $M$, the fragility of polymers usually increases or remains constant. It appears that PIB at low $M$ exhibits unique behavior-a decrease of fragility with increase in molecular weight.

Recently, Qin and McKenna ${ }^{28}$ suggested a correlation between $T_{\mathrm{g}}$ and fragility. This observation is supported by a rather weak trend presented for many polymers. Following this idea, Sokolov et al. ${ }^{27}$ argued that polymers with a strong molecular weight-dependent $T_{\mathrm{g}}$ (e.g., PS and PMMA) exhibit a strong increase in fragility, whereas polymers with a weak molecular weight dependence of $T_{\mathrm{g}}$ (e.g., PDMS) show weak or no increase in fragility. However, the molecular weight dependence of $T_{\mathrm{g}}$ for PIB is normal, notwithstanding its unusual dependence of fragility on molecular weight. Thus, PIB once again fails to conform to trends observed for many other polymers.

To our knowledge, low-molecular weight PIB is the only polymer to exhibit a decrease in fra-

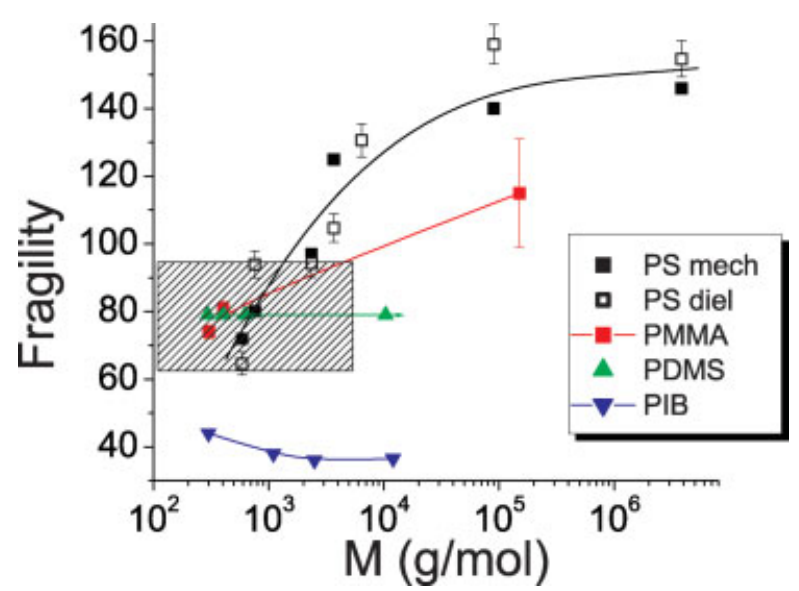

Figure 6. Molecular weight dependence of fragility in various polymers. The shaded area represents the region characteristic of small molecules. The fragility values for PIB are those calculated from dielectric spectroscopy. [Color figure can be viewed in the online issue, which is available at www.interscience.wiley. com.]

Journal of Polymer Science: Part B: Polymer Physics DOI $10.1002 /$ polb 
gility with increasing $M$. PIB is also one of the least fragile polymers. ${ }^{10}$ The reasons for these peculiarities are not understood. Ngai and Roland ${ }^{12}$ proposed that the symmetry of the monomer and the absence of bulky pendant groups play a role in the fragility of polymers through weakened intermolecular cooperativity. According to the coupling model, polymers with smooth symmetric chain structure have weak intermolecular constraints on local motions that leads to lower $m .^{12}$ This concept alone cannot account for the comparison of PIB to PDMS, since the latter has symmetric side groups but a much higher fragility. The failure might be related to the need to consider additional thermodynamic variables, as has been addressed in recent developments of the coupling model. ${ }^{29,30}$

Another possible explanation is the absence of tacticity in PIB chains. Atactic asymmetric polymer chains have many different structures dictated by the stereoconfiguration of the repeat units. This results in many possible conformational states, each with its corresponding configurational energy, leading to a broad distribution of configurational energy minima. A broad distribution of energy minima is usually associated with fragile behavior ${ }^{31,32}$ (although the topology of the energy landscape is also strongly influenced by the heterogeneous molecular packing). Since PIB has a symmetric repeat unit, the distribution of configurational energies might be independent of chain length. ${ }^{33}$ Moreover, it has been established from analysis of $n$-alkanes that the $\mathrm{C}-\mathrm{C}$ rotational energy barrier increases slightly with increase in chain length, from $3.0 \mathrm{kcal} / \mathrm{mol}$ in ethane to $3.6 \mathrm{kcal} / \mathrm{mol}$ in butane. A similar increase of rotational energy barrier can also be expected in PIB. We speculate that the slight increase of rotational barrier with chain length without any increase in the distribution of energy minima might lead to a slight decrease in fragility with molecular weight in PIB. This speculation is supported by the observation that the VFT temperature $T_{0}$ appears to be rather independent of molecular weight (Table 1). Thus, an increase in molecular weight leads to an increase in $T_{\mathrm{g}}$ without significant change in $T_{0}$. This results in an "effective" decrease in fragility with $M$ (eq 4), suggesting the unusual molecular weight dependence of fragility in PIB might be related to the unusual independence of $T_{0}$ on $M$.

An important thermodynamic property of PIB that once again differentiates this material from many other polymers is the fact that different conformational states (trans and gauche) have essentially the same energy. ${ }^{34}$ As a result, changes in temperature do not significantly affect the population of conformational states of the chain molecule. This peculiar property of PIB might also contribute to its unusual behavior.

We need to emphasize that other polymers such as PDMS also have a symmetric structural unit and no tacticity. However, PDMS exhibits commonly observed polymeric behavior and has a much higher fragility. ${ }^{15}$ So, the roles of symmetry and tacticity remain to be fully investigated. In particular, the conformational energies of the PDMS rotameric states differ significantly, so that the molecule changes its equilibrium conformation with temperature. ${ }^{34} \mathrm{Al}$ though PDMS and PIB have the same methyl side groups, their backbones have very different flexibilities. For PDMS the characteristic energy barrier for conformational changes of the backbone is less than $2 \mathrm{~kJ} / \mathrm{mol},{ }^{35}$ whereas methyl group rotation has a barrier $\sim 7 \mathrm{~kJ} / \mathrm{mol}^{36}$ Thus, methyl groups in PDMS can exert steric constraints on the motions of the (otherwise flexible) backbone. In contrast, the methyl groups in PIB have an energy barrier, $\sim 14 \mathrm{~kJ} / \mathrm{mol}^{37}$ which is comparable to the energy barrier for conformational transitions of the backbone, $E \sim$ $18-25 \mathrm{~kJ} / \mathrm{mol}^{35,37}$ This implies that conformational transitions of the PIB backbone occur on a time scale similar to the side group motions, thus coupling their respective dynamics. ${ }^{38}$ Evidently, the relative flexibility of the backbone and pendant groups can exert a significant influence on the fragility of polymers.

This idea agrees with the recent theoretical work $^{14}$ that relates fragility to polymer chain packing: polymers that pack well (e.g., flexible chains without bulky side groups) are expected to be strong, whereas polymers with poor packing (e.g., rigid chains and those with bulky side groups) are expected to be fragile. The packing model does not take into account the chain tacticity, which can also affect packing. A stereoregular polymer should also pack better, since random arrangement of pendant groups would increase disorder in the system. The packing of PIB is known to be extremely high (which contributes to its low air permeability). In particular, Van Krevelen and Hoftyzer ${ }^{39}$ calculated the density of many polymers using the group increment method and compared it with experimentally measured density. They applied this approach to 85 amorphous polymers and found 
satisfactory agreement with experimental data, with the mean deviation between calculated and experimental values being $1.3 \%$. However, in their analysis PIB stands out having the strongest deviation: its measured density $\left(0.913 \mathrm{~g} / \mathrm{cm}^{3}\right)$ is $7 \%$ higher than the calculated value $(0.851 \mathrm{~g} /$ $\mathrm{cm}^{3}$ ). Thus, the packing of PIB appears to be much higher than the expected from model calculations, which provide reasonable results for many other polymers.

We speculate that the similar flexibility of the PIB side groups and backbone and the lack of atacticity result in the extremely tightly packed structure. Usually, packing efficiency decreases with $M$. This frustration in packing, according to the model, ${ }^{14}$ would cause the increase in fragility with $M$. However, PIB may be exceptional, because its packing actually improves with increasing molecular weight. This idea is supported (indirectly) by the observed variations in the fast dynamics [Fig. 2(b)]. A decrease in the QES intensity (relative to the boson peak intensity) is usually ascribed to a decrease in the amplitude of fast conformational fluctuations (rattling in a cage formed by neighboring monomers). ${ }^{24}$ Thus, the observed decrease of $R_{1}$ with molecular weight in PIB is consistent with tighter packing of PIB chains.

\section{CONCLUSIONS}

This analysis demonstrates the decrease in fragility with increasing molecular weight for PIB with $M \leq 2500 \mathrm{~g} / \mathrm{mol}$, that is, in the range where $T_{\mathrm{g}}$ exhibits strong dependence on molecular weight. This behavior is opposite to that commonly observed in polymers and is not predicted by any theory. The deviation from the usual pattern adds to other known peculiarities in the properties of PIB: low gas permeability, one of the lowest fragility among polymers, a segmental relaxation dispersion that is much broader than expected for strong glass-formers, a small difference between the time-temperature shift factors for segmental and chain relaxations, and so forth. These observations suggest that PIB may be the most unusual polymer, although the reasons for its peculiar behavior require further study. The similar flexibility of the backbone and side groups, the absence of conformational effects due to the symmetric structure of the repeat unit and lack of tacticity, and the near equivalence of the energy for different conforma- tional states may all contribute. These factors might give rise to a distribution of configurational energies that is independent of chain length, and thus the anomalous molecular weight dependence of the fragility of PIB. It would be important to discover another polymer with similar properties, to clarify the microscopic origin of the behavior of PIB. At least one conclusion is obvious: PIB is an ironic choice to illustrate the general properties of polymers. However, contrasting the unusual behavior of PIB to other polymers helps to develop a deeper understanding of the regular behavior of polymeric materials.

The authors thank P. G. Santangelo (NRL) for experimental assistance, and C. G. Robertson (Bridgestone Americas) and C. Alba-Simionesco (University Paris Sud) for many helpful and stimulating discussions. The Akron University team thanks the NSF Polymer program for financial support (grant DMR-0605784). M. Paluch acknowledges the financial support of the Committee for Scientific Research, Poland (KBN, Grant No. N202 147 32/4240) and C. M. Roland the support of the Office of Naval Research.

\section{REFERENCES AND NOTES}

1. Marvin, R. S. In Proceedings of the 2nd International Congress on Rheology; Harrison, V. G. W., Ed.; Butterworths: London, 1954; pp 156-164.

2. Ferry, JD. In Viscoelastic Properties of Polymers; Wiley: New York, 1980; p 482.

3. Boyd, R. H.; Pant, P. V. K. Macromolecules 1991, 24, 6325-6331.

4. Plazek, D. J.; Zheng, X. D.; Ngai, K. L. Macromolecules 1992, 25, 4920-4924.

5. Ngai, K. L.; Plazek, D. J. Rubber Chem Tech Rubber Rev 1995, 68, 376-434.

6. Frick, B.; Richter, D.; Trevino, S. Phys A 1993, 201, 88-94.

7. Ngai, K. L.; Plazek, D. J Macromol 1990, 23, 4282-4287.

8. Santangelo, P. G.; Ngai, K. L.; Roland, C. M. Macromolecules 1993, 26, 2682-2687.

9. Sokolov, A. P.; Novikov, V. N.; Kisliuk, A.; Ngai, K. L. Phys Rev B 2001, 63, 172204.

10. Boehmer, R.; Ngai, K. L.; Angell, C. A., Plazek, D. J. J Chem Phys 1993, 99, 4201-4209.

11. Casalini, R.; Roland, C. M.; Capaccioli, S. J Chem Phys 2007, 126, 184903.

12. Ngai, K. L.; Roland, C. M. Macromolecules 1993, 26, 6824-6830.

13. Roland, C. M.; Ngai, K. L. Macromolecules 1996 , 29, 5747-5750.

14. Dudowicz, J.; Freed, K. F.; Douglas, J. F. J Phys Chem B 2005, 109, 21285-21292.

Journal of Polymer Science: Part B: Polymer Physics DOI $10.1002 /$ polb 
15. Ding, Y.; Novikov, V. N.; Sokolov, A. P.; Cailliaux, A.; Dalle-Ferrier, C.; Alba-Simionesco, C.; Frick, B. Macromolecules 2004, 37, 9264-9272.

16. Puskas, J. E.; Lanzendörfer, M. G. Macromolecules 1998, 31, 8684-8690.

17. Kennedy, J. P.; Sivaram, S. J Macromol Sci, 1973, A-7, 969-989.

18. Robertson, C. G.; Santangelo, P. G.; Roland, C. M. J Non-Cryst Solids 2000, 275, 153-159.

19. Puskas, J. E.; Chen, Y.; Kulbaba, K.; Kaszas, G.; Soleymannezhad, A. J Polym Sci Chem 2006, 44, 1770-1776.

20. Roland, C. M.; Paluch, M.; Rzoska, S. J. J Chem Phys 2003, 119, 12439-12441.

21. Saltzman, E. J., Schweizer, K. S. J Chem Phys 2004, 121, 1984-2000.

22. Ngai, K. L.; Sokolov, A. P.; Steffen, W. J Chem Phys 1997, 107, 5268-5272.

23. Sokolov, A. P.; Rd́ossler, E.; Kisliuk, A.; Quitmann, D. Phys Rev Lett 1993, 71, 2062-2065.

24. Novikov, V. N.; Ding, Y.; Sokolov, A. P. Phys Rev E 2005, 71, 061501.

25. Roland, C. M.; Casalini, R. J Chem Phys 2003, 119, 1838-1842.

26. Santangelo, P. G.; Roland, C. M. Macromolecules 1998, 31, 4581-4585.
27. Sokolov, A. P.; Novikov, V. N.; Ding, Y. J Phys: Condens Matter 2007, 19, 205116.

28. Qin, Q.; McKenna, G. B. J Non-Cryst Solids 2006, 352, 2977-2985.

29. Ngai, K. L.; Casalini, R.; Roland, C. M. Macromolecules 2005, 38, 4363-4370.

30. Ngai, K. L. J Non-Cryst Solids 2005, 351, 2635-2642.

31. Zheng, Q.; Durben, D. J.; Wolf, G. H. Angell, C. A. Science 1995, 254, 829-832.

32. Debenedetti, P. G.; Stillinger, F. H. Nature 2001, 410, 259-267.

33. Suter, U. W.; Saiz, E.; Flory, P. J Macromolecules 1983, 16, 1317-1328.

34. Erman, B.; Mark, JE. In Structures and Properties of Rubberlike Networks; Oxford University Press: New York, 1997; pp 126.

35. Somoza, M. M.; Sluch, M. I.; Berg, M. A. Macromolecules 2003, 36, 2721-2732.

36. Mukhopadhyay, R.; Alegra, A.; Colmenero, J.; Frick, B. Macromolecules 1998, 31, 3985-3993.

37. Karatasos, K.; Saija, F.; Ryckaert, J. P. Physica B 2001, 301, 119-125.

38. Karatasos, K.; Ryckaert, J. P.; Ricciardi, R.; Laupretre, F. Macromolecules 2002, 35, 1451-1462.

39. Krevelen, D. W.; Hoftyzer, P. J. J Appl Polym Sci 1969, 13, 871-881. 